\title{
Quantum Stochastic Gradients
}

\author{
Un Cig JI ${ }^{1, *}$ and Nobuaki OBATA ${ }^{2, *}$ \\ ${ }^{1}$ Department of Mathematics, Research Institute of Mathematical Finance, \\ Chungbuk National University, Cheongju 361-763, Korea \\ ${ }^{2}$ Graduate School of Information Sciences, Tohoku University, Sendai 980-8579, Japan
}

Received February 25, 2009; final version accepted May 17, 2009

\begin{abstract}
We obtain regularity properties of the quantum stochastic gradients (creation, annihilation and conservation gradients) by systematic study of their various domains. Accordingly, regularity properties of the (non-adapted) quantum stochastic integrals of Hitsuda-Skorohod type follow by duality.
\end{abstract}

KEYWORDS: Hitsuda-Skorohod integral, quantum stochastic integral, quantum white noise, stochastic gradient, white noise operator

\section{Introduction}

Since the celebrated paper by Hudson-Parthasarathy [7] the quantum stochastic integrals of Itô type have been extensively studied by many authors, see Meyer [17], Parthasarathy [21] for fundamentals and Attal-Lindsay [2] for more recent results. Beyond the Itô type, non-adapted (or non-causal) quantum stochastic integrals have been discussed by Belavkin [3] and Lindsay [15]. In this paper, we propose a functional analytic method (kernel theorem and duality, see e.g., Treves [22]) for further investigation, in particular, towards regularity problems of quantum stochastic integrals. First we introduce new notions of quantum stochastic gradients which are quantum extensions of the classical stochastic gradient. We then define by duality the Hitsuda-Skorohod quantum stochastic integrals. Our approach seems advantageous to systematic study of regularity properties of the quantum stochastic integrals.

In the classical context the Hitsuda-Skorohod integral is defined by means of the adjoint action of the stochastic gradient (also called the Malliavin gradient), see e.g., Kuo [14], Malliavin [16], Nualart [18]. Let us explain in terms of white noise theory $[6,14,19]$. Our consideration is based on the Gelfand triple

$$
(E) \subset \Gamma(H)=\Gamma\left(L^{2}(\mathbb{R})\right) \subset(E)^{*},
$$

where $\Gamma(H)$ is isomorphic to the $L^{2}$-space over the Gaussian space through the Wiener-Itô-Segal isomorphism. We adopt the so-called the Hida-Kubo-Takenaka space of white noise functions, see Section 2. The stochastic gradient $\nabla$ is defined by

$$
\nabla \phi(t)=a_{t} \phi, \quad \phi \in(E), \quad t \in \mathbb{R},
$$

where $a_{t}$ is the annihilation operator at a point $t \in \mathbb{R}$. Then, $\nabla$ is extended to a suitable domain larger than $(E)$, say $\mathbf{D}$, and becomes a continuous linear map from $\mathbf{D}$ into $L^{2}(\mathbb{R}, \Gamma(H))$, see Section 2.3 for more precise description. The adjoint map $\delta$ of $\nabla$ is then a continuous map from $L^{2}(\mathbb{R}, \Gamma(H))$ into $\mathbf{D}^{*}$. We call $\delta(\Psi)$ the Hitsuda-Skorohod integral. In probabilistic interpretation, $\nabla$ maps a random variable to a stochastic process and $\delta$ conversely.

In the quantum context we need to treat processes consisting of unbounded operators in the Fock space $\Gamma(H)$, in particular, the annihilation, creation and conservation processes. The framework (1.1) is convenient to overcome this difficulty. A continuous operator from $(E)$ into $(E)^{*}$ is called a white noise operator and $\mathcal{L}\left((E),(E)^{*}\right)$ denotes the space of such operators. The three basic quantum stochastic processes become $\mathcal{L}\left((E),(E)^{*}\right)$-valued continuous (in fact, infinitely many times differentiable) maps defined on $\mathbb{R}$. Moreover, so is the quantum white noise $\left\{a_{t}, a_{t}^{*}\right\}$. This fact is the basis of white noise approach to quantum stochastic calculus, see e.g., [5, 8-10].

Our discussion relies often on the canonical topological isomorphism

$$
\mathcal{K}: \mathcal{L}\left((E),(E)^{*}\right) \longrightarrow(E)^{*} \otimes(E)^{*}
$$

and its restrictions. Then we can introduce three extensions of the classical gradient, namely,

Mathematics Subject Classifications (2000): primary 81S25; secondary 46F25, 60H40

* Corresponding author. E-mail: uncigji@cbucc.chungbuk.ac.kr; obata@math.is.tohoku.ac.jp 


$$
\begin{aligned}
& \nabla^{+}[\phi \otimes \psi](t)=(\nabla \phi(t)) \otimes \psi, \\
& \nabla^{-}[\phi \otimes \psi](t)=\phi \otimes(\nabla \psi(t)), \\
& \nabla^{0}[\phi \otimes \psi](t)=(\nabla \phi(t)) \otimes(\nabla \psi(t)) .
\end{aligned}
$$

These are formulated as continuous linear maps with suitable domains, see Section 3 for details. Through the canonical isomorphism (1.2) each $\nabla^{\epsilon}$ defines a continuous linear map from a certain space of white noise operators to a space of $L^{2}$-functions with values in white noise operators. We call $\nabla^{+}, \nabla^{-}$and $\nabla^{0}$ the creation, annihilation and conservation gradients, respectively.

The adjoint map of $\nabla^{\epsilon}$, denoted by $\delta^{\epsilon}$, becomes a continuous linear map from a certain space of $L^{2}$-functions with values in white noise operators into a space of white noise operators. These are called the creation, annihilation and conservation integrals, respectively. It will be shown that

$$
\delta^{+}(\Xi) \phi=\delta(\Xi \phi), \quad \delta^{-}(\Xi) \phi=\int_{\mathbb{R}} \Xi(t)(\nabla \phi(t)) d t, \quad \delta^{0}(\Xi) \phi=\delta(\Xi \nabla \phi),
$$

where the right-hand sides are in terms of the classical stochastic gradient and the classical Hitsuda-Skorohod integral. Thus, our definitions of the Hitsuda-Skorohod quantum stochastic integrals coincide with the ones introduced by Belavkin [3] and Lindsay [15] for a common integrand. In fact, their definition starts with the right-hand sides of (1.3) for suitably chosen $\Xi$ and $\phi$. Our approach is more direct thanks to the quantum stochastic gradients acting on white noise operators and is expected to be advantageous to systematic study of regularity properties of quantum stochastic integrals, as is illustrated in Sections 4 and 5.

The basic idea of quantum stochastic gradients were first introduced in the recently published paper [12], where we discussed also an application to quantum martingales. More applications to quantum stochastic integral representations of Fock space operators are found in [13]. In this paper, introducing several different domains of the quantum stochastic gradients, we investigate their regularity properties in detail. Then we obtain the regularity properties of quantum stochastic integrals by duality.

\section{Stochastic Gradient in White Noise Theory}

\subsection{White noise distributions}

We start with the real Gelfand triple:

$$
\varsigma(\mathbb{R}) \subset L_{\mathbb{R}}^{2}(\mathbb{R}, d t) \subset \varsigma^{\prime}(\mathbb{R}),
$$

where $L_{\mathbb{R}}^{2}(\mathbb{R}, d t)$ is the Hilbert space of $\mathbb{R}$-valued square-integrable functions on $\mathbb{R}, \delta(\mathbb{R})$ the space of rapidly decreasing functions, and $\delta^{\prime}(\mathbb{R})$ the space of tempered distributions. The inner product of $L_{\mathbb{R}}^{2}(\mathbb{R}, d t)$ and the canonical bilinear form on $\ell^{\prime}(\mathbb{R}) \times \delta(\mathbb{R})$ are compatible and are denoted by the same symbol $\langle\cdot$,$\rangle . The complexification of (2.1)$ is denoted by

$$
E \subset H=L^{2}(\mathbb{R}) \subset E^{*}
$$

for simplicity. (Throughout this paper $L^{2}(\ldots)$ denotes the complex $L^{2}$-space.) The canonical $\mathbb{C}$-bilinear form on $E^{*} \times E$ is denoted also by $\langle\cdot, \cdot\rangle$. Let $|\cdot|_{0}$ denote the norm of $L^{2}(\mathbb{R})$. Note that $|\xi|_{0}^{2}=\langle\bar{\xi}, \xi\rangle$ for $\xi \in H$.

The topology of $E$ is defined by means of the differential operator $A=1+t^{2}-d^{2} / d t^{2}$ acting in $H$. For each $p \geq 0$ we put $E_{p}=\operatorname{Dom}\left(A^{p}\right)$ which becomes a Hilbert space with norm $|\xi|_{p}=\left|A^{p} \xi\right|_{0}$. Let $E_{-p}$ be the completion of $H$ with respect to the norm $|\xi|_{-p}=\left|A^{-p} \xi\right|_{0}$. Then $E_{-p}$ is identified with the strong dual space of $E_{p}$ with respect to the Hilbert space $H$. We thus obtain a chain of Hilbert spaces:

$$
\cdots \subset E_{p} \subset \cdots \subset H \subset \cdots \subset E_{-p} \subset \cdots .
$$

It is known that

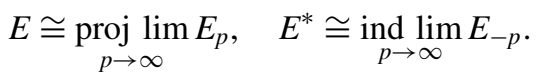

In fact, $E$ is a countably Hilbert nuclear space. There exists an orthonormal basis $\left\{e_{i}\right\}_{i=0}^{\infty} \subset E$ of $H$ such that $A e_{i}=$ $(2 i+2) e_{i}$ for $i=0,1,2, \ldots$. The constant number $\rho=\left\|A^{-1}\right\|_{\mathrm{OP}}=1 / 2$ appears often in norm estimates throughout.

The (Boson) Fock space over $E_{p}$ is defined by

$$
\Gamma\left(E_{p}\right)=\left\{\phi=\left(f_{n}\right)_{n=0}^{\infty} ; f_{n} \in E_{p}^{\hat{\otimes} n},\|\phi\|_{p}^{2}=\sum_{n=0}^{\infty} n !\left|f_{n}\right|_{p}^{2}<\infty\right\},
$$

where $\widehat{\otimes}$ is the symmetric tensor product. Taking the chain of Fock spaces:

$$
\cdots \subset \Gamma\left(E_{p}\right) \subset \cdots \subset \Gamma(H) \subset \cdots \subset \Gamma\left(E_{-p}\right) \subset \cdots
$$


into account, we define

$$
(E)=\underset{p \rightarrow \infty}{\operatorname{proj} \lim } \Gamma\left(E_{p}\right), \quad(E)^{*}=\underset{p \rightarrow \infty}{\operatorname{ind} \lim } \Gamma\left(E_{-p}\right) .
$$

It is known that $(E)$ is a countably Hilbert nuclear space. We thus obtain a complex Gelfand triple:

$$
(E) \subset \Gamma(H) \subset(E)^{*},
$$

which is referred to as the Hida-Kubo-Takenaka space. By definition the topology of $(E)$ is defined by the norms

$$
\|\phi\|_{p}^{2}=\sum_{n=0}^{\infty} n !\left|f_{n}\right|_{p}^{2}, \quad \phi=\left(f_{n}\right), \quad p \in \mathbb{R} .
$$

On the other hand, for each $\Phi \in(E)^{*}$ there exists $p \geq 0$ such that $\Phi \in \Gamma\left(E_{-p}\right)$. In this case, we have

$$
\|\Phi\|_{-p}^{2}=\sum_{n=0}^{\infty} n !\left|F_{n}\right|_{-p}^{2}<\infty, \quad \Phi=\left(F_{n}\right) .
$$

The canonical $\mathbb{C}$-bilinear form on $(E)^{*} \times(E)$ takes the form:

$$
\langle\langle\Phi, \phi\rangle\rangle=\sum_{n=0}^{\infty} n !\left\langle F_{n}, f_{n}\right\rangle, \quad \Phi=\left(F_{n}\right) \in(E)^{*}, \quad \phi=\left(f_{n}\right) \in(E) .
$$

\subsection{White noise operators}

A continuous linear operator from $(E)$ into $(E)^{*}$ is called a white noise operator. The space of white noise operators is denoted by $\mathcal{L}\left((E),(E)^{*}\right)$ and is equipped with the bounded convergence topology. The white noise operators cover a wide class of Fock space operators, for example, $\mathcal{L}((E),(E)), \mathcal{L}\left((E)^{*},(E)\right)$ and $\mathcal{B}(\Gamma(H))$ are subspaces of $\mathcal{L}\left((E),(E)^{*}\right)$, where $\mathcal{B}(\Gamma(H))$ is the space of bounded operators on $\Gamma(H)$.

Since $(E)$ is a nuclear space, applying the kernel theorem (e.g., Treves [22]) we have the canonical isomorphism:

$$
\mathcal{K}: \mathcal{L}\left((E),(E)^{*}\right) \stackrel{\cong}{\longrightarrow}(E)^{*} \otimes(E)^{*},
$$

which is defined by

$$
\langle\langle\Xi \phi, \psi\rangle\rangle=\langle\langle\mathcal{K} \Xi, \psi \otimes \phi\rangle, \quad \phi, \psi \in(E) .
$$

Then, as restrictions of $\mathcal{K}$ we obtain similar isomorphisms such as

$$
\begin{gathered}
\mathcal{L}\left((E)^{*},(E)\right) \cong(E) \otimes(E), \quad \mathcal{L}((E),(E)) \cong(E) \otimes(E)^{*}, \\
\mathcal{L}\left((E)^{*},(E)^{*}\right) \cong(E)^{*} \otimes(E) . \quad \mathcal{L}\left((E)^{*}, \Gamma(H)\right) \cong \Gamma(H) \otimes(E), \quad \text { etc. }
\end{gathered}
$$

It is noted that the Hilbert space tensor product $\Gamma(H) \otimes \Gamma(H)$ is isomorphic to the space of Hilbert-Schmidt operators $\mathcal{L}_{2}(\Gamma(H), \Gamma(H))$.

With each $x \in E^{*}$ we associate the annihilation operator $a(x)$ defined by

$$
a(x): \phi=\left(f_{n}\right)_{n=0}^{\infty} \mapsto\left((n+1) x \otimes_{1} f_{n+1}\right)_{n=0}^{\infty},
$$

where $x \otimes_{1} f_{n}$ stands for the contraction. It is easily verified that $a(x) \in \mathcal{L}((E),(E))$. Its adjoint operator $a^{*}(x) \in$ $\mathcal{L}\left((E)^{*},(E)^{*}\right)$ is called the creation operator and satisfies

$$
\left.a^{*}(x): \phi=\left(f_{n}\right)_{n=0}^{\infty} \mapsto\left(x \hat{\otimes} f_{n-1}\right)_{n=0}^{\infty}, \quad \text { (understanding that } f_{-1}=0\right) .
$$

In particular, for each $t \in \mathbb{R}$ we put

$$
a_{t}=a\left(\delta_{t}\right), \quad a_{t}^{*}=a^{*}\left(\delta_{t}\right) .
$$

These are called respectively the annihilation operator and creation operator at a point $t$. The pair $\left\{a_{t}, a_{t}^{*} ; t \in \mathbb{R}\right\}$ is called the quantum white noise.

\subsection{Classical stochastic gradient}

We first note the following fundamental fact, of which the proof is contained implicitly in [19, Chapter 4].

Lemma 2.1. The map $t \mapsto a_{t}$ is an $\mathcal{L}((E),(E))$-valued rapidly decreasing function, i.e., belong to $8(\mathbb{R}) \otimes$ $\mathcal{L}((E),(E)) \cong \mathcal{L}((E), \wp(\mathbb{R}) \otimes(E)) \cong \wp(\mathbb{R}, \mathcal{L}((E),(E)))$.

Hence, for $\phi \in(E)$ the map $t \mapsto a_{t} \phi$ is an $(E)$-valued rapidly decreasing function. Then the map $\nabla$ defined by

$$
\nabla \phi(t)=a_{t} \phi, \quad \phi \in(E), \quad t \in \mathbb{R}
$$

becomes a continuous linear map from $(E)$ into $\varsigma(\mathbb{R}) \otimes(E) \cong \wp(\mathbb{R},(E)$ ). 
We need to extend the domain of $\nabla$. For $\phi=\left(f_{n}\right) \in(E)^{*}$ we set

$$
\|\phi\|_{\mathbf{D}}^{2}=\sum_{n=0}^{\infty}(n+1) n !\left|f_{n}\right|_{0}^{2} .
$$

Then $\mathbf{D}=\left\{\phi \in(E)^{*} ;\|\phi\|_{\mathbf{D}}<\infty\right\}$ is a subspace of $\Gamma(H)$ and becomes a Hilbert space equipped with the norm $\|\cdot\|_{\mathbf{D}}$. The dual space is identified with $\mathbf{D}^{*}=\left\{\Phi \in(E)^{*} ;\|\Phi\|_{\mathbf{D}^{*}}<\infty\right\}$, where

$$
\|\Phi\|_{\mathbf{D}^{*}}^{2}=\sum_{n=0}^{\infty}(n+1)^{-1} n !\left|F_{n}\right|_{0}^{2}, \quad \Phi=\left(F_{n}\right) \in(E)^{*} .
$$

We then have the inclusion relations:

$$
(E) \subset \mathbf{D} \subset \Gamma(H) \subset \mathbf{D}^{*} \subset(E)^{*} .
$$

We first extend the domain of $\nabla$ to $\mathbf{D}$ by virtue of the following

Lemma 2.2. The map $\nabla$ in (2.3) is extended uniquely to a continuous linear map from $\mathbf{D}$ into $L^{2}(\mathbb{R}) \otimes$ $\Gamma(H) \cong L^{2}(\mathbb{R}, \Gamma(H))$. Denoting the extension by the same symbol, we have

$$
\|\nabla \phi\|_{L^{2}(\mathbb{R}, \Gamma(H))} \leq\|\phi\|_{\mathbf{D}}, \quad \phi \in \mathbf{D} .
$$

The proof is straightforward, see e.g., Kuo [14, Chapter 9]. A further extension is possible.

Lemma 2.3. The map $\nabla$ in (2.3) is extended uniquely to a continuous linear map from $\Gamma(H)$ into $L^{2}(\mathbb{R}) \otimes$ $\mathbf{D}^{*} \cong L^{2}\left(\mathbb{R}, \mathbf{D}^{*}\right)$. Denoting the extension by the same symbol, we have

$$
\|\nabla \phi\|_{L^{2}\left(\mathbb{R}, \mathbf{D}^{*}\right)} \leq\|\phi\|_{\Gamma(H)}, \quad \phi \in \Gamma(H) .
$$

Proof. By definition,

$$
\|\nabla \phi\|_{L^{2}\left(\mathbb{R}, \mathbf{D}^{*}\right)}^{2}=\int_{\mathbb{R}}\left\|a_{t} \phi\right\|_{\mathbf{D}^{*}}^{2} d t, \quad \phi=\left(f_{n}\right) \in(E) .
$$

It follows from (2.4) that

$$
\left\|a_{t} \phi\right\|_{\mathbf{D}^{*}}^{2}=\sum_{n=0}^{\infty}(n+1)^{-1} n !\left|(n+1) f_{n+1}(t, \cdot)\right|_{0}^{2}=\sum_{n=0}^{\infty}(n+1) !\left|f_{n+1}(t, \cdot)\right|_{0}^{2} .
$$

Hence (2.6) becomes

$$
\|\nabla \phi\|_{L^{2}\left(\mathbb{R}, \mathbf{D}^{*}\right)}^{2}=\sum_{n=0}^{\infty}(n+1) ! \int_{\mathbb{R}}\left|f_{n+1}(t, \cdot)\right|_{0}^{2} d t=\sum_{n=0}^{\infty}(n+1) !\left|f_{n+1}\right|_{0}^{2} \leq\|\phi\|_{\Gamma(H)}^{2},
$$

which proves (2.5).

Summing up the above argument, we come to the following diagram:

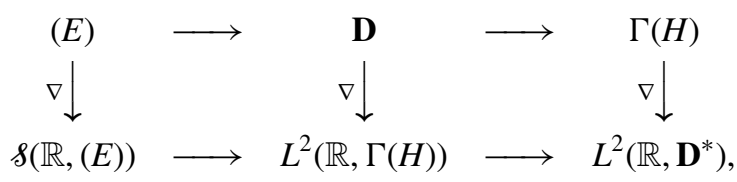

where the right arrows are continuous injections (inclusions) and the down arrows are continuous linear maps, which differ in domains but are denoted by the symbol $\nabla$. We refer to $\nabla$ as the (classical) stochastic gradient. The middle $\nabla$ in (2.7) appears often in literatures, see e.g., Kuo [14], Malliavin [16], Nualart [18].

Proposition 2.4. Let $\zeta \in L^{2}(\mathbb{R})$. Then $a(\zeta) \in \mathcal{L}(\mathbf{D}, \Gamma(H))$ and we have

$$
a(\zeta) \phi=\int_{\mathbb{R}} \zeta(t) \nabla \phi(t) d t, \quad \phi \in \mathbf{D}
$$

Moreover, it holds that

$$
\langle\langle a(\zeta) \phi, \psi\rangle\rangle=\langle\langle\nabla \phi, \zeta \otimes \psi\rangle\rangle, \quad \phi \in \mathbf{D}, \quad \psi \in \Gamma(H) .
$$

Also, $a(\zeta) \in \mathcal{L}\left(\Gamma(H), \mathbf{D}^{*}\right)$ and similar identities as in (2.8) and (2.9) hold.

Proof. It is straightforward to check (2.8) for exponential vectors $\phi_{\xi}$ with $\xi \in E$. Observing that both sides are continuous in $\phi$ with respect to the topology of $\mathbf{D}$, we get the result. The proof of (2.9) is then obvious. 


\section{Quantum Stochastic Gradients}

\subsection{Creation gradient}

We will define the creation gradient as a continuous linear map acting on white noise operators. Several domains will be introduced.

(Case 1) $\mathscr{L}((E), \mathbf{D})$ and $\mathscr{L}\left((E)^{*}, \mathbf{D}\right)$. We first consider $\tilde{\nabla}^{+}$defined by

$$
\tilde{\nabla}^{+}: \mathbf{D} \otimes(E)^{*} \stackrel{\nabla \otimes I}{\longrightarrow} L^{2}(\mathbb{R}, \Gamma(H)) \otimes(E)^{*} \stackrel{\cong}{\longrightarrow} L^{2}\left(\mathbb{R}, \Gamma(H) \otimes(E)^{*}\right),
$$

where the first arrow is defined by the middle version of stochastic gradient (2.7) and the second one needs clarification. It is known that

$$
L^{2}(\mathbb{R}, \Gamma(H)) \otimes(E)^{*} \cong \text { ind } \lim _{p \rightarrow \infty} L^{2}(\mathbb{R}, \Gamma(H)) \otimes \Gamma\left(E_{-p}\right) .
$$

On the other hand, in view of the isomorphisms among Hilbert spaces:

$$
\begin{aligned}
L^{2}(\mathbb{R}, \Gamma(H)) \otimes \Gamma\left(E_{-p}\right) & \cong\left(L^{2}(\mathbb{R}) \otimes \Gamma(H)\right) \otimes \Gamma\left(E_{-p}\right) \\
& \cong L^{2}(\mathbb{R}) \otimes\left(\Gamma(H) \otimes \Gamma\left(E_{-p}\right)\right) \\
& \cong L^{2}\left(\mathbb{R}, \Gamma(H) \otimes \Gamma\left(E_{-p}\right)\right),
\end{aligned}
$$

where the tensor products are all in the sense of Hilbert spaces, we define

$$
L^{2}\left(\mathbb{R}, \Gamma(H) \otimes(E)^{*}\right)=\operatorname{ind} \lim _{p \rightarrow \infty} L^{2}\left(\mathbb{R}, \Gamma(H) \otimes \Gamma\left(E_{-p}\right)\right) .
$$

Then, combining (3.2) and (3.3), we obtain the isomorphism in (3.1). Recall that $\mathcal{K}$ in (2.2) gives rise to isomorphisms: $\mathscr{L}((E), \mathbf{D}) \rightarrow \mathbf{D} \otimes(E)^{*} \quad$ and $\quad \mathcal{L}((E), \Gamma(H)) \rightarrow \Gamma(H) \otimes(E)^{*}$. The latter naturally induces an isomorphism $L^{2}(\mathbb{R}, \mathcal{L}((E), \Gamma(H))) \rightarrow L^{2}\left(\mathbb{R}, \Gamma(H) \otimes(E)^{*}\right)$, which is denoted also by $\mathcal{K}$, i.e., $\mathcal{K}(\Xi)(t)=\mathcal{K}(\Xi(t))$. With these notations we define

$$
\nabla^{+}=\mathcal{K}^{-1} \circ \tilde{\nabla}^{+} \circ \mathcal{K}
$$

In other words, $\nabla^{+}$is the composition of continuous linear maps:

$$
\nabla^{+}: \mathcal{L}((E), \mathbf{D}) \stackrel{\cong}{\longrightarrow} \mathbf{D} \otimes(E)^{*} \stackrel{\tilde{\nabla}^{+}}{\longrightarrow} L^{2}\left(\mathbb{R}, \Gamma(H) \otimes(E)^{*}\right) \stackrel{\cong}{\longrightarrow} L^{2}(\mathbb{R}, \mathcal{L}((E), \Gamma(H))) .
$$

We call $\nabla^{+}$the creation gradient.

Next consider $\mathcal{L}\left((E)^{*}, \mathbf{D}\right)$, which is regarded as a subspace of $\mathcal{L}((E), \mathbf{D})$. The creation gradient $\nabla^{+}$restricted to $\mathscr{L}\left((E)^{*}, \mathbf{D}\right)$ is the composition of continuous linear maps:

$$
\begin{aligned}
\nabla^{+}: \mathcal{L}\left((E)^{*}, \mathbf{D}\right) & \stackrel{\cong}{\longrightarrow} \mathbf{D} \otimes(E) \stackrel{\nabla \otimes I}{\longrightarrow} L^{2}(\mathbb{R}, \Gamma(H)) \otimes(E) \\
& \cong L^{2}(\mathbb{R}, \Gamma(H) \otimes(E)) \stackrel{\cong}{\longrightarrow} L^{2}\left(\mathbb{R}, \mathcal{L}\left((E)^{*}, \Gamma(H)\right)\right),
\end{aligned}
$$

where $L^{2}(\mathbb{R}, \Gamma(H) \otimes(E))$ is defined by

$$
L^{2}(\mathbb{R}, \Gamma(H) \otimes(E))=\underset{p \rightarrow \infty}{\operatorname{proj} \lim } L^{2}\left(\mathbb{R}, \Gamma(H) \otimes \Gamma\left(E_{p}\right)\right) .
$$

For the norm estimates of $\nabla^{+}$we first prove the following

Theorem 3.1. Let $p \in \mathbb{R}$. For any $\Xi \in \mathscr{L}_{2}\left(\Gamma\left(E_{p}\right)\right.$, D) its creation gradient $\nabla^{+} \Xi$ belongs to $L^{2}\left(\mathbb{R}, \mathcal{L}_{2}\left(\Gamma\left(E_{p}\right), \Gamma(H)\right)\right)$ and

$$
\int_{\mathbb{R}}\left\|\nabla^{+} \Xi(t)\right\|_{\mathscr{L}_{2}\left(\Gamma\left(E_{p}\right), \Gamma(H)\right)}^{2} d t \leq\|\Xi\|_{\mathscr{L}_{2}\left(\Gamma\left(E_{p}\right), \mathbf{D}\right)}^{2} .
$$

In particular,

$$
\nabla^{+}: \mathscr{L}_{2}\left(\Gamma\left(E_{p}\right), \mathbf{D}\right) \rightarrow L^{2}\left(\mathbb{R}, \mathcal{L}_{2}\left(\Gamma\left(E_{p}\right), \Gamma(H)\right)\right)
$$

is a contraction.

Proof. We see that $\nabla^{+}$restricted to $\mathcal{L}_{2}\left(\Gamma\left(E_{p}\right), \mathbf{D}\right)$ is the composition of maps:

$$
\begin{aligned}
\nabla^{+}: \mathscr{L}_{2}\left(\Gamma\left(E_{p}\right), \mathbf{D}\right) & \stackrel{\cong}{\longrightarrow} \mathbf{D} \otimes \Gamma\left(E_{-p}\right) \stackrel{\nabla \otimes I}{\longrightarrow} L^{2}(\mathbb{R}, \Gamma(H)) \otimes \Gamma\left(E_{-p}\right) \\
& \cong L^{2}\left(\mathbb{R}, \Gamma(H) \otimes \Gamma\left(E_{-p}\right)\right) \stackrel{\cong}{\longrightarrow} L^{2}\left(\mathbb{R}, \mathcal{L}_{2}\left(\Gamma\left(E_{p}\right), \Gamma(H)\right)\right),
\end{aligned}
$$

where the isomorphisms $\cong$ are all unitary isomorphisms between Hilbert spaces. On the other hand, $\nabla: \mathbf{D} \rightarrow$ $L^{2}(\mathbb{R}, \Gamma(H))$ is a contraction by Lemma 2.2 , so is the above $\nabla \otimes I$. Then (3.4) follows. 
Corollary 3.2. For any $\Xi \in \mathcal{L}_{2}(\Gamma(H)$, D) we have

$$
\int_{\mathbb{R}}\left\|\nabla^{+} \Xi(t)\right\|_{\mathcal{L}_{2}(\Gamma(H), \Gamma(H))}^{2} d t \leq\|\Xi\|_{\mathcal{L}_{2}(\Gamma(H), \mathbf{D})}^{2} .
$$

In particular, $\nabla^{+} \Xi(t)$ is a Hilbert-Schmidt operator on $\Gamma(H)$ for a.e. $t \in \mathbb{R}$.

The above discussion on the creation gradient is summarized into the following diagram:

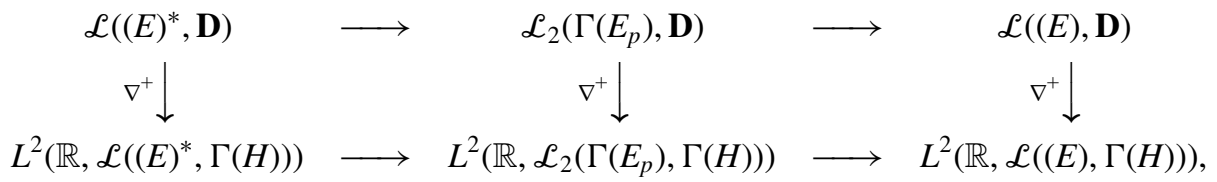

where $p$ runs over $\mathbb{R}$ and the right arrows are continuous injections (inclusions). Note that

$$
\begin{aligned}
& \mathcal{L}((E), \mathbf{D}) \cong \mathbf{D} \otimes(E)^{*} \cong \operatorname{ind}_{p \rightarrow \infty} \mathbf{l i m} \otimes \Gamma\left(E_{-p}\right) \cong \operatorname{ind}_{p \rightarrow \infty} \lim _{2} \mathcal{L}_{2}\left(\Gamma\left(E_{p}\right), \mathbf{D}\right), \\
& \mathcal{L}\left((E)^{*}, \mathbf{D}\right) \cong \mathbf{D} \otimes(E) \cong \underset{p \rightarrow \infty}{\operatorname{proj} \lim } \mathbf{D} \otimes \Gamma\left(E_{p}\right) \cong \underset{p \rightarrow \infty}{\operatorname{proj} \lim } \mathcal{L}_{2}\left(\Gamma\left(E_{-p}\right), \mathbf{D}\right) .
\end{aligned}
$$

Hence, a white noise operator $\Xi$ belongs to $\mathcal{L}((E), \mathbf{D})$ if and only if there exists $p \geq 0$ such that $\Xi \in \mathcal{L}_{2}\left(\Gamma\left(E_{p}\right)\right.$, D). Similarly, $\Xi$ belongs to $\mathcal{L}\left((E)^{*}, \mathbf{D}\right)$ if and only if $\Xi \in \mathcal{L}_{2}\left(\Gamma\left(E_{-p}\right), \mathbf{D}\right)$ for all $p \geq 0$. Thus, the norm estimate of $\nabla^{+}$in (3.5) is obtained from Theorem 3.1.

(Case 2) $\mathcal{L}((E), \Gamma(H))$ and $\mathcal{L}\left((E)^{*}, \Gamma(H)\right)$. We define

$$
\begin{aligned}
\nabla^{+}: \mathcal{L}((E), \Gamma(H)) & \stackrel{\cong}{\longrightarrow} \Gamma(H) \otimes(E)^{*} \stackrel{\nabla \otimes I}{\longrightarrow} L^{2}\left(\mathbb{R}, \mathbf{D}^{*}\right) \otimes(E)^{*} \\
& \cong \\
\longrightarrow & L^{2}\left(\mathbb{R}, \mathbf{D}^{*} \otimes(E)^{*}\right) \stackrel{\cong}{\longrightarrow} L^{2}\left(\mathbb{R}, \mathcal{L}\left((E), \mathbf{D}^{*}\right)\right),
\end{aligned}
$$

where the classical gradient appears as a continuous linear map (in fact, contraction) from $\Gamma(H)$ into $L^{2}\left(\mathbb{R}, \mathbf{D}^{*}\right)$, see the third version of (2.7). Then the argument is totally parallel to Case 1 and we obtain the following

Theorem 3.3. Let $p \in \mathbb{R}$. For any $\Xi \in \mathcal{L}_{2}\left(\Gamma\left(E_{p}\right), \Gamma(H)\right)$ its creation gradient $\nabla^{+} \Xi$ belongs to $L^{2}\left(\mathbb{R}, \mathcal{L}_{2}\left(\Gamma\left(E_{p}\right), \mathbf{D}^{*}\right)\right)$ and

$$
\int_{\mathbb{R}}\left\|\nabla^{+} \Xi(t)\right\|_{\mathscr{L}_{2}\left(\Gamma\left(E_{p}\right), \mathbf{D}^{*}\right)}^{2} d t \leq\|\Xi\|_{\mathscr{L}_{2}\left(\Gamma\left(E_{p}\right), \Gamma(H)\right)}^{2}
$$

In particular,

$$
\nabla^{+}: \mathcal{L}_{2}\left(\Gamma\left(E_{p}\right), \Gamma(H)\right) \rightarrow L^{2}\left(\mathbb{R}, \mathcal{L}_{2}\left(\Gamma\left(E_{p}\right), \mathbf{D}^{*}\right)\right)
$$

is a contraction.

We also note the following diagram:

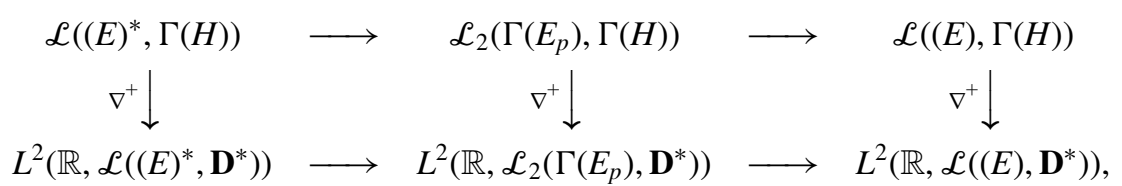

where $p$ runs over $\mathbb{R}$ and

$$
\begin{aligned}
& \mathcal{L}((E), \Gamma(H)) \cong \text { ind } \lim _{p \rightarrow \infty} \mathcal{L}_{2}\left(\Gamma\left(E_{p}\right), \Gamma(H)\right), \\
& \mathcal{L}\left((E)^{*}, \Gamma(H)\right) \cong \underset{p \rightarrow \infty}{\operatorname{proj} \lim } \mathcal{L}_{2}\left(\Gamma\left(E_{-p}\right), \Gamma(H)\right) .
\end{aligned}
$$

Finally we mention a role of the creation gradient, see Proposition 2.4 for the classical case.

Theorem 3.4. Let $\Xi$ be a member of one of the domains of the creation gradient in (3.5) and (3.6). Then, for $\zeta \in H=L^{2}(\mathbb{R})$ the composition $a(\zeta) \Xi$ is defined as a continuous operator and admits the integral expression:

$$
a(\zeta) \Xi=\int_{\mathbb{R}} \zeta(t) \nabla^{+} \Xi(t) d t
$$

Proof. We prove the assertion for $\Xi \in \mathcal{L}_{2}\left(\Gamma\left(E_{p}\right), \mathbf{D}\right)$. Other cases are similar. It is straightforward to see that the composition $a(\zeta) \Xi$ is defined and belongs to $\mathcal{L}((E), \Gamma(H))$. We show (3.7). Let $\phi \in(E)$ and $\psi \in \Gamma(H)$. Then $t \mapsto\left\langle\left\langle\nabla^{+} \Xi(t) \phi, \psi\right\rangle\right\rangle$ belongs to $L^{2}(\mathbb{R})$. In fact, 


$$
\left|\left\langle\left\langle\nabla^{+} \Xi(t) \phi, \psi\right\rangle\right\rangle\right| \leq\left\|\nabla^{+} \Xi(t) \phi\right\|_{0}\|\psi\|_{0} \leq\left\|\nabla^{+} \Xi(t)\right\|_{\mathcal{L}_{2}\left(\Gamma\left(E_{p}\right), \Gamma(H)\right)}\|\phi\|_{p}\|\psi\|_{0}
$$

and by Theorem 3.1 we obtain

$$
\begin{aligned}
\int_{\mathbb{R}}\left|\left\langle\left\langle\nabla^{+} \Xi(t) \phi, \psi\right\rangle\right\rangle\right|^{2} d t & \leq\|\phi\|_{p}^{2}\|\psi\|_{0}^{2} \int_{\mathbb{R}}\left\|\nabla^{+} \Xi(t)\right\|_{\mathcal{L}_{2}\left(\Gamma\left(E_{p}\right), \Gamma(H)\right)}^{2} d t \\
& \leq\|\phi\|_{p}^{2}\|\psi\|_{0}^{2}\|\Xi\|_{\mathcal{L}_{2}\left(\Gamma\left(E_{p}\right), \mathbf{D}\right)}^{2}<\infty .
\end{aligned}
$$

Then, for any $\zeta \in L^{2}(\mathbb{R})$ we have

$$
\begin{aligned}
\int_{\mathbb{R}} \zeta(t)\left\langle\left\langle\nabla^{+} \Xi(t) \phi, \psi\right\rangle\right\rangle d t & =\int_{\mathbb{R}}\left\langle\left\langle\nabla^{+} \Xi(t) \phi, \zeta(t) \psi\right\rangle\right\rangle d t=\left\langle\left\langle\mathcal{K} \nabla^{+} \Xi, \zeta \otimes \psi \otimes \phi\right\rangle\right\rangle \\
& =\langle(\nabla \otimes I) \mathcal{K} \Xi, \zeta \otimes \psi \otimes \phi\rangle=\left\langle\left\langle\mathcal{K} \Xi, \nabla^{*}(\zeta \otimes \psi) \otimes \phi\right\rangle\right\rangle \\
& =\left\langle\Xi \Xi \phi, \nabla^{*}(\zeta \otimes \psi)\right\rangle=\langle\langle\nabla \Xi \phi, \zeta \otimes \psi\rangle\rangle .
\end{aligned}
$$

We see from Proposition 2.4 that the last expression becomes $\langle\langle a(\zeta) \Xi \Phi, \psi\rangle\rangle$. Consequently,

$$
\int_{\mathbb{R}} \zeta(t)\left\langle\left\langle\nabla^{+} \Xi(t) \phi, \psi\right\rangle\right\rangle d t=\langle\langle a(\zeta) \Xi \phi, \psi\rangle\rangle
$$

which proves the assertion.

Remark We can define the creation gradient in a more general context. Let $\mathscr{H}$ be an arbitrary Hilbert space and $\mathscr{H}^{*}$ its dual space. Cases 1 and 2 are for $\mathscr{H}=\Gamma\left(E_{p}\right)$. We define a continuous linear map $\nabla^{+}: \mathscr{L}_{2}(\mathscr{H}, \mathbf{D}) \rightarrow$ $L^{2}\left(\mathbb{R}, \mathscr{L}_{2}(\mathscr{H}, \Gamma(H))\right)$ by

$$
\begin{aligned}
\nabla^{+}: \mathscr{L}_{2}(\mathcal{H}, \mathbf{D}) & \stackrel{\cong}{\longrightarrow} \mathbf{D} \otimes \mathscr{H}^{*} \stackrel{\nabla \otimes I}{\longrightarrow} L^{2}(\mathbb{R}, \Gamma(H)) \otimes \mathcal{H}^{*} \\
& \cong L^{2}\left(\mathbb{R}, \Gamma(H) \otimes \mathcal{H}^{*}\right) \stackrel{\cong}{\longrightarrow} L^{2}\left(\mathbb{R}, \mathcal{L}_{2}(\mathcal{H}, \Gamma(H))\right) .
\end{aligned}
$$

Then, as in Theorem 3.1 we see that

$$
\int_{\mathbb{R}}\left\|\nabla^{+} \Xi(t)\right\|_{\mathscr{L}_{2}(\mathscr{H}, \Gamma(H))}^{2} d t \leq\|\Xi\|_{\mathscr{L}_{2}(\mathscr{H}, \mathbf{D})}^{2} .
$$

Similarly, a continuous linear map $\left.\nabla^{+}: \mathscr{L}_{2}(\mathscr{H}, \Gamma(H)) \rightarrow L^{2}\left(\mathbb{R}, \mathcal{L}_{2}\left(\mathscr{H}, \mathbf{D}^{*}\right)\right)\right)$ is defined and satisfies

$$
\int_{\mathbb{R}}\left\|\nabla^{+} \Xi(t)\right\|_{\mathscr{L}_{2}\left(\mathscr{H}, \mathbf{D}^{*}\right)}^{2} d t \leq\|\Xi\|_{\mathcal{L}_{2}(\mathscr{H}, \Gamma(H))}^{2} .
$$

\subsection{Annihilation gradient}

Recall that one of the domains of the creation gradient $\nabla^{+}$is $\mathcal{L}((E), \mathbf{D})$, see (3.5). We consider the space of adjoint operators, i.e., $\mathcal{L}\left(\mathbf{D}^{*},(E)^{*}\right)$, and define $\nabla^{-}$by composition as follows:

$$
\begin{aligned}
\nabla^{-}: \mathcal{L}\left(\mathbf{D}^{*},(E)^{*}\right) & \cong(E)^{*} \otimes \mathbf{D} \stackrel{I \otimes \nabla}{\longrightarrow}(E)^{*} \otimes L^{2}(\mathbb{R}, \Gamma(H)) \\
& \cong L^{2}\left(\mathbb{R},(E)^{*} \otimes \Gamma(H)\right) \stackrel{\cong}{\longrightarrow} L^{2}\left(\mathbb{R}, \mathcal{L}\left(\Gamma(H),(E)^{*}\right)\right),
\end{aligned}
$$

where $L^{2}\left(\mathbb{R},(E)^{*} \otimes \Gamma(H)\right)$ is defined by

$$
L^{2}\left(\mathbb{R},(E)^{*} \otimes \Gamma(H)\right)=\operatorname{ind} \lim _{p \rightarrow \infty} L^{2}\left(\mathbb{R}, \Gamma\left(E_{-p}\right) \otimes \Gamma(H)\right) .
$$

We call $\nabla^{-}$the annihilation gradient. Consideration similar to (3.5) yields

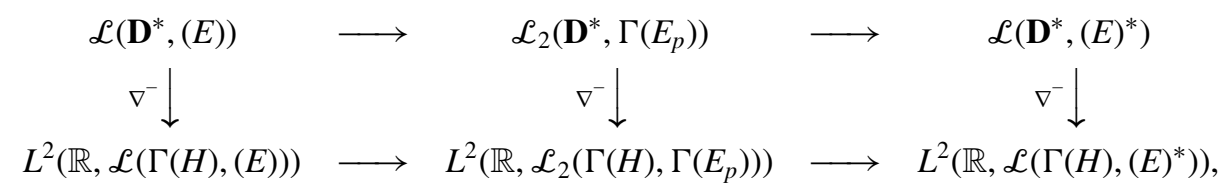

where

$$
\mathcal{L}\left(\mathbf{D}^{*},(E)\right) \cong \underset{p \rightarrow \infty}{\operatorname{proj} \lim } \mathcal{L}_{2}\left(\mathbf{D}^{*}, \Gamma\left(E_{p}\right)\right), \quad \mathcal{L}\left(\mathbf{D}^{*},(E)^{*}\right) \cong \operatorname{ind}_{p \rightarrow \infty} \lim _{2} \mathcal{L}_{2}\left(\mathbf{D}^{*}, \Gamma\left(E_{-p}\right)\right) .
$$

For the norm estimate of the annihilation gradient we have the following result, of which the proof is similar to that of Theorem 3.1.

Theorem 3.5. Let $p \in \mathbb{R}$. For any $\Xi \in \mathscr{L}_{2}\left(\mathbf{D}^{*}, \Gamma\left(E_{p}\right)\right)$ its annihilation gradient $\nabla^{-} \Xi$ belongs to $L^{2}\left(\mathbb{R}, \mathscr{L}_{2}\left(\Gamma(H), \Gamma\left(E_{p}\right)\right)\right)$ and 


$$
\int_{\mathbb{R}}\left\|\nabla^{-} \Xi(t)\right\|_{\mathcal{L}_{2}\left(\Gamma(H), \Gamma\left(E_{p}\right)\right)}^{2} d t \leq\|\Xi\|_{\mathcal{L}_{2}\left(\mathbf{D}^{*}, \Gamma\left(E_{p}\right)\right)}^{2}
$$

Corollary 3.6. For any $\Xi \in \mathcal{L}_{2}\left(\mathbf{D}^{*}, \Gamma(H)\right)$ its annihilation gradient $\nabla^{-} \Xi$ belongs to $L^{2}\left(\mathbb{R}, \mathcal{L}_{2}(\Gamma(H), \Gamma(H))\right)$ and

$$
\int_{\mathbb{R}}\left\|\nabla^{-} \Xi(t)\right\|_{\mathcal{L}_{2}(\Gamma(H), \Gamma(H))}^{2} d t \leq\|\Xi\|_{\mathcal{L}_{2}\left(\mathbf{D}^{*}, \Gamma(H)\right)}^{2} .
$$

In particular, $\nabla^{-} \Xi(t)$ is a Hilbert-Schmidt operator on $\Gamma(H)$ for a.e. $t \in \mathbb{R}$.

Repeating similar consideration, we obtain the following diagram:

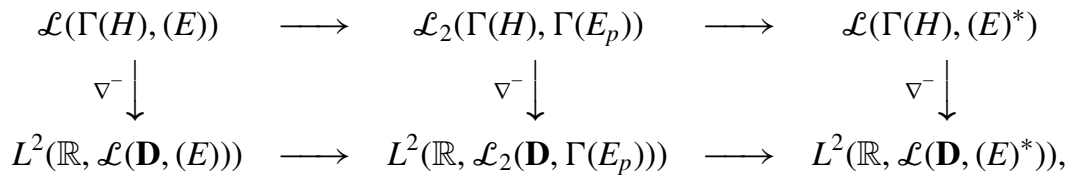

which corresponds to (3.6). The norm estimates are similar to (3.9) and (3.10).

Theorem 3.7. Let $\Xi$ be a member of one of the domains of the annihilation gradient in (3.8) and (3.11). Then, for $\zeta \in H=L^{2}(\mathbb{R})$ the composition $\Xi a(\zeta)^{*}$ is defined as a continuous operator and admits the integral expression:

$$
\Xi a^{*}(\zeta)=\int_{\mathbb{R}} \zeta(t) \nabla^{-} \Xi(t) d t
$$

Moreover,

$$
\nabla^{-} \Xi(t)=\left(\nabla^{+} \Xi^{*}(t)\right)^{*} \text { for a.e. } t \in \mathbb{R} \text {. }
$$

Proof. The proof of (3.12) is similar to that of Theorem 3.4, while (3.13) follows from

$$
\Xi a^{*}(\zeta)=\left(a(\zeta) \Xi^{*}\right)^{*}=\left(\int_{\mathbb{R}} \zeta(t) \nabla^{+} \Xi^{*}(t) d t\right)^{*}=\int_{\mathbb{R}} \zeta(t)\left(\nabla^{+} \Xi^{*}(t)\right)^{*} d t,
$$

which is easily justified.

\subsection{Conservation gradient}

We need the "diagonalized" tensor product $\nabla \oslash \nabla$ of the stochastic gradients. We begin with the following.

Lemma 3.8. For any $p \geq 0$ and $q>0$ with $p+q>5 / 12$ there exists a constant $C(p, q)>0$ such that

$$
\sup _{t \in \mathbb{R}}\|\nabla \psi(t)\|_{p}^{2} \leq C(p, q)\|\psi\|_{p+q}^{2}, \quad \psi \in(E) .
$$

Proof. We first note that

$$
\sup _{t \in \mathbb{R}}\left|\delta_{t}\right|_{-r}<\infty, \quad r>\frac{5}{12}
$$

which is verified by mimicking the argument in [20, Appendix]. Then, for any pair of $p, q$ satisfying the assumption, we have a finite constant $C(p, q)$ defined by

$$
C(p, q)=\max \left\{\rho^{2 q n}(n+1)\left|\delta_{t}\right|_{-(p+q)}^{2} ; t \in \mathbb{R}, n=0,1,2, \ldots\right\}<\infty .
$$

Now for $\psi=\left(g_{n}\right) \in(E)$ we have $\nabla \psi(t)=a_{t} \psi$ so that

$$
\begin{aligned}
\|\nabla \psi(t)\|_{p}^{2} & =\sum_{n=0}^{\infty} n !\left|(n+1) \delta_{t} \otimes_{1} g_{n+1}\right|_{p}^{2} \\
& \leq \sum_{n=0}^{\infty} \rho^{2 q n}(n+1)\left|\delta_{t}\right|_{-(p+q)}^{2}(n+1) !\left|g_{n+1}\right|_{p+q}^{2},
\end{aligned}
$$

where a basic formula for norm inequalities is used, see [19, Appendix C]. Taking (3.15) into account, we obtain

$$
\|\nabla \psi(t)\|_{p}^{2} \leq C(p, q)\|\psi\|_{p+q}^{2}, \quad t \in \mathbb{R},
$$

which completes the proof. 
For each $\phi \in \mathbf{D}$ and $\psi \in(E)$ we define

$$
[(\nabla \oslash \nabla) \phi \otimes \psi](t)=\nabla \phi(t) \otimes \nabla \psi(t), \quad \text { for a.e. } t \in \mathbb{R} .
$$

Then, with the help of Lemmas 2.2 and 3.8 one can show easily that

$$
\int_{\mathbb{R}}\|[(\nabla \oslash \nabla) \phi \otimes \psi](t)\|_{\Gamma(H) \otimes \Gamma\left(E_{p}\right)}^{2} d t \leq C(p, q)\|\phi\|_{\mathbf{D}}^{2}\|\psi\|_{p+q}^{2}
$$

Therefore, $\nabla \oslash \nabla: \mathbf{D} \otimes_{\pi} \Gamma\left(E_{p+q}\right) \rightarrow L^{2}\left(\mathbb{R}, \Gamma(H) \otimes \Gamma\left(E_{p}\right)\right)$ is continuous, where $\otimes_{\pi}$ is the $\pi$-tensor product (not the Hilbert space tensor product). Since $\mathbf{D} \otimes(E)=\mathbf{D} \otimes_{\pi}(E) \rightarrow \mathbf{D} \otimes_{\pi} \Gamma\left(E_{p+q}\right)$ is a continuous injection, we see that

$$
\nabla \oslash \nabla: \mathbf{D} \otimes(E) \rightarrow L^{2}(\mathbb{R}, \Gamma(H) \otimes(E))
$$

is a continuous linear map. The conservation gradient is now defined by compositions of continuous linear maps:

$$
\nabla^{0}: \mathcal{L}\left((E)^{*}, \mathbf{D}\right) \stackrel{\cong}{\longrightarrow} \mathbf{D} \otimes(E) \stackrel{\nabla \oslash \nabla}{\longrightarrow} L^{2}(\mathbb{R}, \Gamma(H) \otimes(E)) \stackrel{\cong}{\longrightarrow} L^{2}\left(\mathbb{R}, \mathcal{L}\left((E)^{*}, \Gamma(H)\right)\right),
$$

where

$$
L^{2}(\mathbb{R}, \Gamma(H) \otimes(E))=\underset{p \rightarrow \infty}{\operatorname{proj} \lim _{p}} L^{2}\left(\mathbb{R}, \Gamma(H) \otimes \Gamma\left(E_{p}\right)\right) \cong\left(L^{2}(\mathbb{R}) \otimes \Gamma(H)\right) \otimes(E) .
$$

In a similar manner,

$$
\nabla^{0}: \mathcal{L}\left((E)^{*}, \Gamma(H)\right) \stackrel{\cong}{\longrightarrow} \Gamma(H) \otimes(E) \stackrel{\nabla \oslash \nabla}{\longrightarrow} L^{2}\left(\mathbb{R}, \mathbf{D}^{*} \otimes(E)\right) \stackrel{\cong}{\longrightarrow} L^{2}\left(\mathbb{R}, \mathcal{L}\left((E)^{*}, \mathbf{D}^{*}\right)\right)
$$

becomes also a continuous linear map. Summing up,

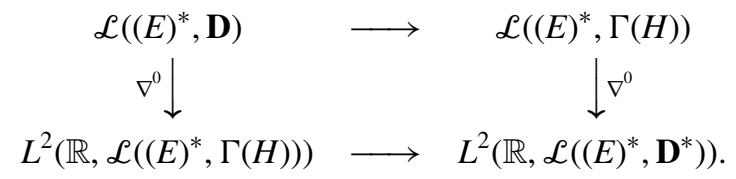

We call $\nabla^{0}$ the conservation gradient.

\section{Hitsuda-Skorohod Quantum Stochastic Integrals}

\subsection{The Hitsuda-Skorohod integral}

The classical stochastic integral of Hitsuda-Skorohod type is defined by means of the adjoint action of the classical stochastic gradient. Taking the adjoint maps in the diagram (2.7), we come to the following

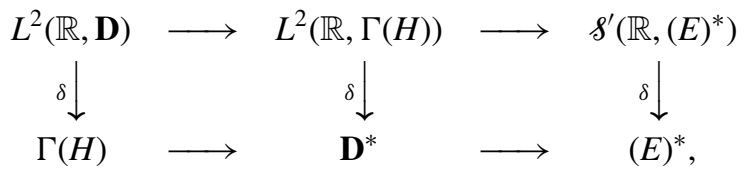

where $\delta=\nabla^{*}$. We call $\delta(\Psi)$ the Hitsuda-Skorohod integral. By definition it holds that

$$
\langle\langle\delta(\Psi), \phi\rangle\rangle=\int_{\mathbb{R}}\langle\langle\Psi(t), \nabla \phi(t)\rangle\rangle d t,
$$

for a suitable pair $\Psi$ and $\phi$.

The quantum stochastic integrals of Hitsuda-Skorohod type are defined in the same spirit, where the quantum stochastic gradients play a role.

\subsection{Creation integral}

The creation integral $\delta^{+}$is by definition the adjoint map of the creation gradient $\nabla^{+}$. Taking the adjoint map of (3.5), we have

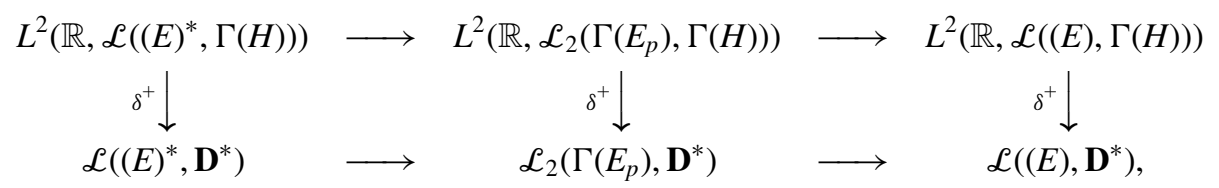

where $p$ runs over $\mathbb{R}$. Similarly from (3.6) we obtain

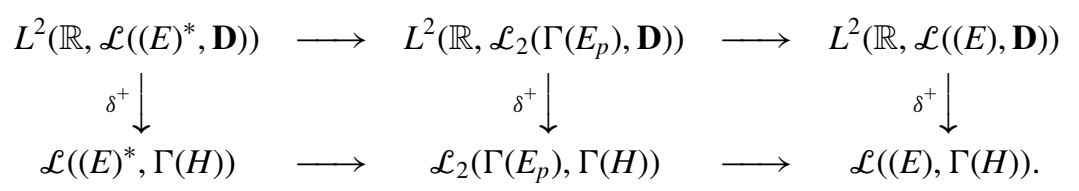


The norm estimates of $\delta^{+}$follow immediately from those of $\nabla^{+}$.

$$
\begin{aligned}
&\left\|\delta^{+}(\Xi)\right\|_{\mathcal{L}_{2}\left(\Gamma\left(E_{p}\right), \mathbf{D}^{*}\right)}^{2} \leq \int_{\mathbb{R}}\|\Xi(t)\|_{\mathcal{L}_{2}\left(\Gamma\left(E_{p}\right), \Gamma(H)\right)}^{2} d t, \\
&\left\|\delta^{+}(\Xi)\right\|_{\mathscr{L}_{2}\left(\Gamma\left(E_{p}\right), \Gamma(H)\right)}^{2} \leq \int_{\mathbb{R}}\|\Xi(t)\|_{\mathcal{L}_{2}\left(\Gamma\left(E_{p}\right), \mathbf{D}\right)}^{2} d t .
\end{aligned}
$$

In particular, putting $p=0$ in (4.5), we obtain

Theorem 4.1. For any $\Xi \in L^{2}\left(\mathbb{R}, \mathcal{L}_{2}(\Gamma(H), \mathbf{D})\right)$ the creation integral $\delta^{+}(\Xi)$ is a Hilbert-Schmidt operator on $\Gamma(H)$.

Theorem 4.2. Let $\Xi$ be a member of one of the domains of the creation integral in (4.2) and (4.3). Then it holds that

$$
\left\langle\left\langle\delta^{+}(\Xi) \phi, \psi\right\rangle\right\rangle=\int_{\mathbb{R}}\langle\langle\Xi(t) \phi, \nabla \psi(t)\rangle\rangle d t
$$

for a suitable pair $\phi, \psi$. Therefore, denoting $(\Xi \phi)(t)=\Xi(t) \phi$ we have

$$
\delta^{+}(\Xi) \phi=\delta(\Xi \phi), \quad \phi \in(E) .
$$

Proof. In order to fix the idea we assume that $\Xi \in L^{2}(\mathbb{R}, \mathcal{L}((E), \Gamma(H)))$ and prove (4.6) for $\phi \in(E)$ and $\psi \in \mathbf{D}$. Recall that $\nabla^{+}=\mathcal{K}^{-1} \circ \tilde{\nabla}^{+} \circ \mathcal{K}$, where $\tilde{\nabla}^{+}: \mathbf{D} \otimes(E) \rightarrow L^{2}(\mathbb{R}, \Gamma(H) \otimes(E))$ is defined in (3.1). Taking the adjoint, we have $\delta^{+}=\mathcal{K}^{-1} \circ \tilde{\delta}^{+} \circ \mathcal{K}$, where $\tilde{\delta}^{+}: L^{2}\left(\mathbb{R}, \Gamma(H) \otimes(E)^{*}\right) \rightarrow \mathbf{D}^{*} \otimes(E)^{*}$ be the adjoint map of $\tilde{\nabla}^{+}$. With these notations we have

$$
\begin{aligned}
\left\langle\left\langle\delta^{+}(\Xi) \phi, \psi\right\rangle\right\rangle & =\left\langle\left\langle\mathcal{K}\left(\delta^{+}(\Xi)\right), \psi \otimes \phi\right\rangle\right\rangle=\left\langle\left\langle\tilde{\delta}^{+}(\mathcal{K}(\Xi)), \psi \otimes \phi\right\rangle\right\rangle \\
& =\left\langle\left\langle\mathcal{K} \Xi, \tilde{\nabla}^{+}(\psi \otimes \phi)\right\rangle\right\rangle=\langle\langle\mathcal{K} \Xi,(\nabla \psi) \otimes \phi\rangle\rangle \\
& =\int_{\mathbb{R}}\langle\langle\mathcal{K} \Xi(t), \nabla \psi(t) \otimes \phi\rangle\rangle d t=\int_{\mathbb{R}}\langle\langle\Xi(t) \phi, \nabla \psi(t)\rangle\rangle d t .
\end{aligned}
$$

The last integral is the Hitsuda-Skorohod integral, see (4.1). Thus,

$$
\left\langle\left\langle\delta^{+}(\Xi) \phi, \psi\right\rangle\right\rangle=\int_{\mathbb{R}}\langle\langle\Xi(t) \phi, \nabla \psi(t)\rangle\rangle d t=\int_{\mathbb{R}}\langle\langle(\Xi \phi)(t), \nabla \psi(t)\rangle\rangle d t=\langle\langle\delta(\Xi \phi), \psi\rangle\rangle,
$$

which proves (4.7).

As for a criterion for $\delta^{+}(\Xi)$ being a bounded operator on $\Gamma(H)$, we only show the following

Theorem 4.3. Let $\Xi$ be a member of one of the domains of the creation integral in (4.2) and (4.3). Assume that there exist a dense subspace $\& \subset \Gamma(H)$ and a constant number $C \geq 0$ such that

$$
\int_{\mathbb{R}}\|\Xi(t) \phi\|_{\mathbf{D}}^{2} d t \leq C\|\phi\|_{0}^{2}, \quad \phi \in \mathcal{E} .
$$

Then the creation integral $\delta^{+}(\Xi)$ is a bounded operator from $\Gamma(H)$ into itself.

Proof. For any $\phi \in \mathcal{E}$ and $\psi \in \Gamma(H)$, we see from (4.6) that

$$
\left|\left\langle\left\langle\delta^{+}(\Xi) \phi, \psi\right\rangle\right\rangle\right| \leq \int_{\mathbb{R}}|\langle\langle\Xi(t) \phi, \nabla \psi(t)\rangle\rangle| d t \leq \int_{\mathbb{R}}\|\Xi(t) \phi\|_{\mathbf{D}}\|\nabla \psi(t)\|_{\mathbf{D}^{*}} d t
$$

Then, by assumption (4.8) and the contraction property of the classical gradient (Lemma 2.3) we obtain

$$
\left|\left\langle\delta^{+}(\Xi) \phi, \psi\right\rangle\right|^{2} \leq \int_{\mathbb{R}}\|\Xi(t) \phi\|_{\mathbf{D}}^{2} d t \int_{\mathbb{R}}\|\nabla \psi(t)\|_{\mathbf{D}^{*}}^{2} d t \leq C\|\phi\|_{0}^{2}\|\psi\|_{0}^{2} .
$$

Hence $\delta^{+}(\Xi)$ is a bounded operator on $\Gamma(H)$.

Corollary 4.4. For any $\Xi \in L^{2}(\mathbb{R}, \mathcal{L}(\Gamma(H), \mathbf{D}))$ the creation integral $\delta^{+}(\Xi)$ is a bounded operator on $\Gamma(H)$.

Proof. By assumption we have

$$
\int_{\mathbb{R}}\|\Xi(t)\|_{\mathscr{L}(\Gamma(H), \mathbf{D})}^{2} d t<\infty
$$

where the integrand is the square of the operator norm. Hence, for $\phi \in \Gamma(H)$ we have

$$
\int_{\mathbb{R}}\|\Xi(t) \phi\|_{\mathbf{D}}^{2} d t \leq\|\phi\|_{0}^{2} \int_{\mathbb{R}}\|\Xi(t)\|_{\mathcal{L}(\Gamma(H), \mathbf{D})}^{2} d t .
$$

Therefore, the condition in Theorem 4.3 is satisfied. 


\subsection{Annihilation integral}

The annihilation integral $\delta^{-}$is defined to be the adjoint map of the annihilation gradient. From (3.8) we obtain

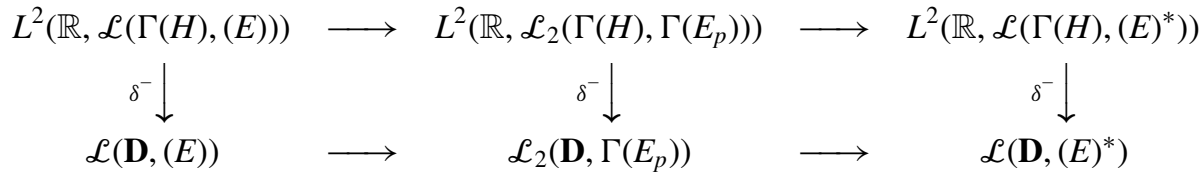

and from (3.11) we obtain

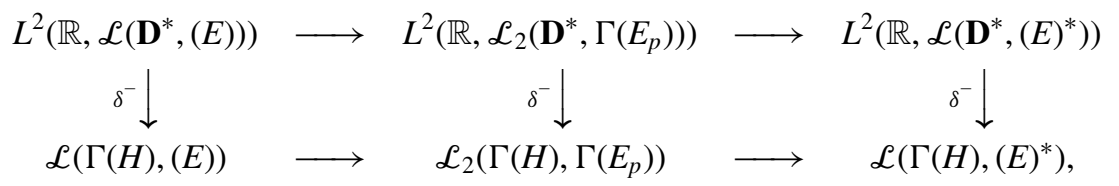

where $p$ runs over $\mathbb{R}$. The norm estimates of $\delta^{-}$follow immediately from those of $\nabla^{-}$and are similar to (4.4) and (4.5) as follows:

$$
\begin{aligned}
\left\|\delta^{-}(\Xi)\right\|_{\mathscr{L}_{2}\left(\mathbf{D}, \Gamma\left(E_{p}\right)\right)}^{2} \leq & \int_{\mathbb{R}}\|\Xi(t)\|_{\mathcal{L}_{2}\left(\Gamma(H), \Gamma\left(E_{p}\right)\right)}^{2} d t, \\
\left\|\delta^{-}(\Xi)\right\|_{\mathscr{L}_{2}\left(\Gamma(H), \Gamma\left(E_{p}\right)\right)}^{2} & \leq \int_{\mathbb{R}}\|\Xi(t)\|_{\mathscr{L}_{2}\left(\mathbf{D}^{*}, \Gamma\left(E_{p}\right)\right)}^{2} d t .
\end{aligned}
$$

In particular, putting $p=0$ in (4.11), we obtain

Theorem 4.5. For any $\Xi \in L^{2}\left(\mathbb{R}, \mathcal{L}_{2}\left(\mathbf{D}^{*}, \Gamma(H)\right)\right)$ the annihilation integral $\delta^{-}(\Xi)$ is a Hilbert-Schmidt operator on $\Gamma(H)$.

In a similar fashion as in Theorem 4.2 we have the following

Theorem 4.6. Let $\Xi$ be a member of one of the domains of the annihilation integral in (4.9) and (4.10). Then it holds that

$$
\left\langle\left\langle\delta^{-}(\Xi) \phi, \psi\right\rangle\right\rangle=\int_{\mathbb{R}}\langle\langle\Xi(t)(\nabla \phi(t)), \psi\rangle\rangle d t
$$

for a suitable pair $\phi, \psi$. Therefore,

$$
\delta^{-}(\Xi) \phi=\int_{\mathbb{R}} \Xi(t)(\nabla \phi(t)) d t
$$

Corollary 4.7. Let $\Xi$ be a member of one of the domains of the annihilation integral in (4.9) and (4.10). Then it holds that

$$
\left(\delta^{-}(\Xi)\right)^{*}=\delta^{+}\left(\Xi^{*}\right)
$$

For the proof we need only to compare (4.6) and (4.12). By virtue of the relation (4.14) one can derive from Theorem 4.3 and Corollary 4.4 a condition for $\delta^{-}(\Xi)$ being a bounded operator on $\Gamma(H)$.

\subsection{Conservation integral}

We defined the conservation gradient $\nabla^{0}$ with two different domains in (3.16). The conservation integral is defined to be their adjoint maps, i.e.,

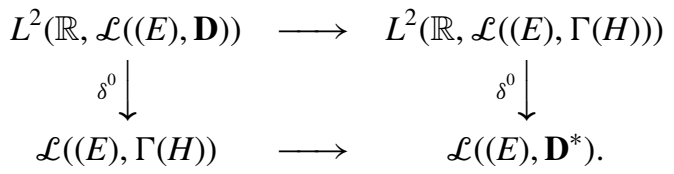

In a similar fashion as in Theorems 4.2 and 4.6 we have the following

Theorem 4.8. Let $\Xi$ be a member of one of the domains of the conservation integral in (4.15). Then it holds that

$$
\left\langle\left\langle\delta^{0}(\Xi) \phi, \psi\right\rangle\right\rangle=\int_{\mathbb{R}}\langle\langle\Xi(t) \nabla \phi(t), \nabla \psi(t)\rangle\rangle d t
$$

for a suitable pair $\phi, \psi$. Therefore, 


$$
\delta^{0}(\Xi) \phi=\delta(\Xi \nabla \phi)
$$

where $\Xi \nabla \phi$ is defined by $\Xi \nabla \phi(t)=\Xi(t)(\nabla \phi(t))$.

We only mention the following criterion for a conservation integral being a bounded operator on $\Gamma(H)$. The proof is similar to that of Theorem 4.3.

Theorem 4.9. Let $\Xi$ be a member of one of the domains of the conservation integral in (4.15). Assume that there exist a dense subspace $\& \subset \Gamma(H)$ and a constant number $C \geq 0$ such that

$$
\int_{\mathbb{R}}\|\Xi(t)(\nabla \phi(t))\|_{\mathbf{D}}^{2} d t \leq C\|\phi\|_{0}^{2}, \quad \phi \in \mathcal{E}
$$

Then the conservation integral $\delta^{0}(\Xi)$ is a bounded operator on $\Gamma(H)$.

Remark We see from (4.7), (4.13) and (4.16) that our definitions of the Hitsuda-Skorohod quantum stochastic integrals coincide with the ones introduced by Belavkin [3] and Lindsay [15] for a common integrand. In fact, their definition starts with the right-hand sides of (4.7), (4.13) and (4.16) for suitably chosen $\Xi$ and $\phi$. Our definition is more direct thanks to the quantum stochastic gradients acting on white noise operators.

\section{Further Extensions}

\subsection{Admissible rigging of Fock space}

We introduce a new family of norms in $\Gamma(H)$. For $p \in \mathbb{R}$ we set

$$
\|\phi\|_{p}^{2}=\sum_{n=0}^{\infty} n ! e^{2 p n}\left|f_{n}\right|_{0}^{2}, \quad \phi=\left(f_{n}\right) \in \Gamma(H) .
$$

For $p \geq 0$ we define $g_{p}=\left\{\phi=\left(f_{n}\right) \in \Gamma(H) ;\|\phi\|_{p}<\infty\right\}$ and $g_{-p}$ to be the completion of $\Gamma(H)$ with respect to $\|\cdot\| \|_{-p}$. Having thus obtained a chain of Hilbert spaces:

$$
\cdots \subset g_{p} \subset \cdots \subset g_{0}=\Gamma(H) \subset \cdots \subset g_{-p} \subset \cdots,
$$

we define

$$
g=\underset{p \rightarrow \infty}{\operatorname{proj} \lim } g_{p}, \quad g^{*}=\underset{p \rightarrow \infty}{\operatorname{ind} \lim } g_{-p}
$$

Note that $g$ is a countable Hilbert space but not a nuclear space. Also note the continuous inclusions:

$$
(E) \subset g \subset \Gamma(H) \subset g^{*} \subset(E)^{*} .
$$

Then $g$ and $g^{*}$ are mutually dual spaces. The canonical $\mathbb{C}$-bilinear form on $g^{*} \times \mathcal{g}$ is denoted by $\langle\langle\cdot, \cdot\rangle\rangle$ again. A white noise operator is called admissible if it belongs to $\mathcal{L}\left(\mathcal{g}, \mathcal{G}^{*}\right)$, which is regarded as a subspace of $\mathcal{L}\left((E),(E)^{*}\right)$.

The spaces $g$ and $g^{*}$ were introduced by Belavkin [3] and have appeared in several contexts, see e.g., [1], [4], [15]. An admissible white noise operator was introduced by Ji-Obata [11] and has been studied along with quantum martingales by Ji [8].

\subsection{Extension of quantum stochastic gradients}

It is easily verified by definition that $g_{p} \subset \mathbf{D}$ for all $p>0$. In particular,

$$
g \subset \mathbf{D} \subset \Gamma(H) \subset \mathbf{D}^{*} \subset g^{*} .
$$

Having defined in Section 2.3 the classical stochastic gradient with domains $\mathbf{D}$ and $\Gamma(H)$, we now extend its domain to $g^{*}$. However, as will be seen below, this extension is no longer a contraction.

Let $p \in \mathbb{R}$. For any $\phi=\left(f_{n}\right)_{n=0}^{\infty} \in g_{p}$ we set

$$
\nabla \phi(t)=\left((n+1) f_{n+1}(t, \cdot)\right)_{n=0}^{\infty},
$$

which is defined for a.e. $t \in \mathbb{R}$ since $f_{n}$ is a symmetric $L^{2}$-function on $\mathbb{R}^{n}$. Then for any $r>0$ we have

$$
\begin{aligned}
\int_{\mathbb{R}}\|\nabla \phi(t)\|_{p}^{2} d t & =\sum_{n=0}^{\infty} n ! e^{2 p n} \int_{\mathbb{R}}\left|(n+1) f_{n+1}(t, \cdot)\right|_{0}^{2} d t \\
& =\sum_{n=0}^{\infty}(n+1) e^{-2 r(n+1)-2 p}(n+1) ! e^{2(p+r)(n+1)}\left|f_{n+1}\right|_{0}^{2} \\
& \leq K(p, r)\|\phi\|_{p+r}^{2}, \quad K(p, r)=\sup _{n} n e^{-2 r n-2 p} .
\end{aligned}
$$


Therefore the stochastic gradient $\nabla$ is a continuous linear map from $g_{p+r}$ into $L^{2}\left(\mathbb{R}, g_{p}\right)$ for any $p \in \mathbb{R}$ and $r>0$. Hence, setting

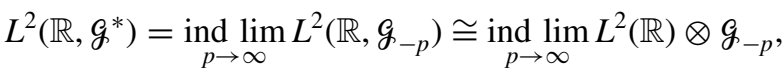

$$
\begin{aligned}
& L^{2}(\mathbb{R}, \mathcal{g})=\underset{p \rightarrow \infty}{\operatorname{proj} \lim } L^{2}\left(\mathbb{R}, g_{p}\right) \cong \underset{p \rightarrow \infty}{\operatorname{proj} \lim } L^{2}(\mathbb{R}) \otimes g_{p},
\end{aligned}
$$

we see that both $\nabla: \mathcal{g} \rightarrow L^{2}(\mathbb{R}, \mathcal{g})$ and $\nabla: g^{*} \rightarrow L^{2}\left(\mathbb{R}, \mathcal{G}^{*}\right)$ are continuous linear maps. In any case we call $\nabla$ the (classical) stochastic gradient.

By similar argument as in Section 3 we can define the quantum stochastic gradients with new domains. For example, the creation gradient is formulated as continuous maps:

$$
\nabla^{+}: \begin{aligned}
& \mathcal{L}\left((E), \mathcal{g}^{*}\right) \rightarrow L^{2}\left(\mathbb{R}, \mathcal{L}\left((E), \mathcal{g}^{*}\right)\right), \\
& \mathcal{L}\left((E)^{*}, \mathcal{g}\right) \rightarrow L^{2}\left(\mathbb{R}, \mathcal{L}\left((E)^{*}, \mathcal{g}\right)\right),
\end{aligned}
$$

and the annihilation gradient as

$$
\nabla^{-}: \begin{aligned}
& \mathcal{L}\left(\mathcal{g},(E)^{*}\right) \rightarrow L^{2}\left(\mathbb{R}, \mathcal{L}\left(\mathcal{G},(E)^{*}\right)\right), \\
& \mathcal{L}\left(\mathcal{G}^{*},(E)\right) \rightarrow L^{2}\left(\mathbb{R}, \mathcal{L}\left(\mathcal{G}^{*},(E)\right)\right) .
\end{aligned}
$$

As for the conservation gradient we consider the diagonalized tensor product $\nabla \oslash \nabla$. First we note by (3.14) and (5.1) that for each $\phi \in \mathcal{G}$ and $\psi \in(E)$,

$$
\int_{\mathbb{R}}\|[(\nabla \oslash \nabla) \phi \otimes \psi](t)\|_{q_{q} \otimes \Gamma\left(E_{p}\right)}^{2} d t \leq K(q, r) C(p, s)\|\phi\|_{q+r}^{2}\|\psi\|_{p+s}^{2},
$$

for any $p \geq 0, q \in \mathbb{R}, r>0$ and $s>0$ with $p+s>5 / 12$. Hence $\nabla \oslash \nabla$ is a continuous linear map from $g_{q+r} \otimes_{\pi}$ $\Gamma\left(E_{p+s}\right)$ into $L^{2}\left(\mathbb{R}, g_{q} \otimes \Gamma\left(E_{p}\right)\right)$. Consequently, the conservation gradient

$$
\nabla^{0}: \mathcal{L}\left((E)^{*}, \mathcal{g}\right) \rightarrow L^{2}\left(\mathbb{R}, \mathcal{L}\left((E)^{*}, \mathcal{g}\right)\right)
$$

becomes a continuous linear map.

\subsection{Extension of quantum stochastic integrals}

The Hitsuda-Skorohod quantum stochastic integrals are defined to be the adjoint maps of quantum stochastic gradients. We have freedom to choose their domains. Among others, in view of (5.2) we obtain the creation integral as

$$
\delta^{+}: \begin{aligned}
& L^{2}\left(\mathbb{R}, \mathcal{L}\left((E)^{*}, \mathcal{G}\right)\right) \rightarrow \mathcal{L}\left((E)^{*}, \mathcal{G}\right), \\
& L^{2}\left(\mathbb{R}, \mathcal{L}\left((E), \mathcal{G}^{*}\right)\right) \rightarrow \mathcal{L}\left((E), \mathcal{G}^{*}\right) .
\end{aligned}
$$

Similarly from (5.3) we have the annihilation integral:

$$
\delta^{-}: \begin{aligned}
& L^{2}\left(\mathbb{R}, \mathcal{L}\left(\mathcal{G}^{*},(E)\right)\right) \rightarrow \mathcal{L}\left(\mathcal{g},(E)^{*}\right), \\
& L^{2}\left(\mathbb{R}, \mathcal{L}\left(\mathcal{G},(E)^{*}\right)\right) \rightarrow \mathcal{L}\left(\mathcal{G},(E)^{*}\right),
\end{aligned}
$$

and from (5.4) the conservation integral:

$$
\delta^{0}: L^{2}\left(\mathbb{R}, \mathcal{L}\left((E), g^{*}\right)\right) \rightarrow \mathcal{L}\left((E), g^{*}\right) .
$$

Although we have another choice of domains in this line, the argument is parallel and omitted.

\subsection{White noise operators}

More generally, we can prove that the stochastic gradients $\nabla \otimes I, I \otimes \nabla$ and $\nabla \oslash \nabla$ are continuous linear maps from $(E) \otimes(E)$ into $\&(\mathbb{R},(E) \otimes(E))$. Therefore, the quantum stochastic integral

$$
\delta^{\epsilon}: \delta^{\prime}\left(\mathbb{R}, \mathcal{L}\left((E),(E)^{*}\right)\right) \rightarrow \mathcal{L}\left((E),(E)^{*}\right)
$$

is a continuous map, where $\epsilon=+,-, 0$. An element of $\mathcal{f}^{\prime}\left(\mathbb{R}, \mathcal{L}\left((E),(E)^{*}\right)\right) \cong \mathcal{L}\left((E),(E)^{*}\right) \otimes \mathcal{f}^{\prime}(\mathbb{R})$ is called a generalized quantum stochastic process, see [20].

\subsection{The classical-quantum correspondence}

It is well known $[9,19]$ that every $\Phi \in(E)^{*}$ gives rise to a white noise operator $M_{\Phi} \in \mathscr{L}\left((E),(E)^{*}\right)$ by multiplication. More precisely, $M_{\Phi}$ is characterized by

$$
\left\langle\left\langle M_{\Phi} \phi, \psi\right\rangle\right\rangle=\langle\langle\Phi, \phi \psi\rangle\rangle, \quad \phi, \psi \in(E),
$$

where $\phi \psi$ is the pointwise product defined through the Wiener-Itô-Segal isomorphism. Let $\phi_{0}=(1,0,0, \ldots) \in(E)$ be the vacuum vector. It then holds that $M_{\Phi} \phi_{0}=\Phi$. 
Lemma 5.1. $M_{\Phi} \in \mathcal{L}\left((E), \mathcal{g}^{*}\right)$ for any $\Phi \in \mathcal{g}^{*}$.

Proof. Choose $q \in \mathbb{R}$ such that $\Phi \in g_{q}$ and write $\Phi=\left(F_{m}\right)$. Let $\phi=\left(g_{n}\right) \in(E)$. Then $M_{\Phi} \phi=\left(h_{l}\right)$ is given by

$$
h_{l}=\sum_{m+n=l} \sum_{k=0}^{\infty} k !\left(\begin{array}{c}
m+k \\
k
\end{array}\right)\left(\begin{array}{c}
n+k \\
k
\end{array}\right) F_{m+k} \widehat{\otimes}_{k} g_{n+k},
$$

see [19, Section 3.5]. Then we have

$$
\begin{aligned}
\sqrt{l !}\left|h_{l}\right|_{0} \leq & \sum_{m+n=l}\left(\begin{array}{c}
m+n \\
m
\end{array}\right)^{1 / 2} 2^{(m+n) / 2} e^{-q m} \rho^{r n} \\
& \times \sum_{k=0}^{\infty}\left(2 \rho^{r} e^{-q}\right)^{k} e^{q(m+k)} \sqrt{(m+k) !} \sqrt{(n+k) !}\left|F_{m+k}\right|_{0}\left|g_{n+k}\right|_{r} .
\end{aligned}
$$

By the Cauchy-Schwarz inequality, the last expression is bounded by

$$
\begin{aligned}
& \leq \sum_{m+n=l}\left(\begin{array}{c}
m+n \\
m
\end{array}\right)^{1 / 2} 2^{(m+n) / 2} e^{-q m} \rho^{r n}\|\Phi\|_{q}\|\phi\|_{r} \\
& \leq\left(\frac{1}{1-\epsilon}\right)^{1 / 2}\left\{\sum_{m+n=l}\left(\begin{array}{c}
m+n \\
m
\end{array}\right)\left(2 e^{-2 q}\right)^{m}\left(\frac{2 \rho^{2 r}}{\epsilon}\right)^{n}\right\}^{1 / 2}\|\Phi\|_{q}\|\phi\|_{r}
\end{aligned}
$$

for any $r>0$ with $2 \rho^{r} e^{-q} \leq 1$ and for any $0<\epsilon<1$. Therefore, for any $p<q$ with $2 e^{2(p-q)}+2 \rho^{2 r} e^{2 p} / \epsilon<1$ we have

$$
\begin{aligned}
\left\|M_{\Phi} \phi\right\|_{p}^{2} & \leq \frac{1}{1-\epsilon} \sum_{l=0}^{\infty}\left\{\sum_{m+n=l}\left(\begin{array}{c}
m+n \\
m
\end{array}\right)\left(2 e^{2(p-q)}\right)^{m}\left(\frac{2 \rho^{2 r} e^{2 p}}{\epsilon}\right)^{n}\right\}\|\Phi\|_{q}^{2}\|\phi\|_{r}^{2} \\
& \leq \frac{1}{1-\epsilon} \sum_{l=0}^{\infty}\left(2 e^{2(p-q)}+\frac{2 \rho^{2 r} e^{2 p}}{\epsilon}\right)^{l}\|\Phi\|_{q}^{2}\|\phi\|_{r}^{2},
\end{aligned}
$$

where the infinite series converges for a suitable choice of parameters. More precisely, for any $p<q, r>0$ and $0<\epsilon<1$ satisfying

$$
\max \left\{2 \rho^{r} e^{-q}, 2 e^{2(p-q)}+\frac{2 \rho^{2 r} e^{2 p}}{\epsilon}\right\}<1,
$$

the infinite series in (5.5) converges and we conclude that $M_{\Phi} \in \mathcal{L}\left(\Gamma\left(E_{r}\right), g_{p}\right)$.

Theorem 5.2. Let $\Phi \in g^{*}$. For any $\zeta \in H$ we have

$$
\int_{\mathbb{R}} \zeta(t)\left[\left(\nabla^{+} M_{\Phi}\right) \phi_{0}\right](t) d t=a(\zeta) \Phi
$$

Proof. It is easily shown by argument similar to the proof of Theorem 3.4 that

$$
a(\zeta) M_{\Phi}=\int_{\mathbb{R}} \zeta(t) \nabla^{+} M_{\Phi}(t) d t
$$

Then, taking the actions on the vacuum vector $\phi_{0}$ we obtain

$$
a(\zeta) \Phi=a(\zeta) M_{\Phi} \phi_{0}=\int_{\mathbb{R}} \zeta(t)\left(\nabla^{+} M_{\Phi}(t)\right) \phi_{0} d t=\int_{\mathbb{R}} \zeta(t)\left[\left(\nabla^{+} M_{\Phi}\right) \phi_{0}\right](t) d t,
$$

where the last equality is by notation.

Theorem 5.3. Let $\Phi \in g^{*}$. For any $\zeta \in H$ we have

$$
\int_{\mathbb{R}} \zeta(t)\left[\left(\nabla^{-} M_{\Phi}\right) \phi_{0}\right](t) d t=\left(a(\zeta)+a^{*}(\zeta)\right) \Phi
$$

Proof. It is proved in a similar fashion as in the proof of Theorem 3.7 that

$$
M_{\Phi} a^{*}(\zeta)=\int_{\mathbb{R}} \zeta(t) \nabla^{-} M_{\Phi}(t) d t
$$

Then we have

$$
M_{\Phi} a^{*}(\zeta) \phi_{0}=\int_{\mathbb{R}} \zeta(t) \nabla^{-} M_{\Phi}(t) \phi_{0} d t=\int_{\mathbb{R}} \zeta(t)\left[\left(\nabla^{-} M_{\Phi}\right) \phi_{0}\right](t) d t
$$


where the last equality is by notation. On the other hand,

$$
M_{\Phi} a^{*}(\zeta) \phi_{0}=M_{a^{*}(\zeta) \phi_{0}} \Phi=\left(a(\zeta)+a^{*}(\zeta)\right) \Phi
$$

where the second equality follows from $\left\langle\left\langle a^{*}(\zeta) \phi_{0}, \phi_{\xi} \phi_{\eta}\right\rangle\right\rangle=\left\langle\left\langle\left(a(\zeta)+a^{*}(\zeta)\right) \phi_{\xi}, \phi_{\eta}\right\rangle\right\rangle$. The assertion follows by combining (5.6) and (5.7).

Theorem 5.4. Let $\Phi \in L^{2}\left(\mathbb{R}, \mathcal{G}^{*}\right)$ and $M_{\Phi}(t)=M_{\Phi(t)}$. Then $M_{\Phi} \in L^{2}\left(\mathbb{R}, \mathcal{L}\left((E), \mathcal{g}^{*}\right)\right)$ and it holds that

$$
\delta^{+}\left(M_{\Phi}\right) \phi_{0}=\delta(\Phi), \quad \delta^{-}\left(M_{\Phi}\right) \phi_{0}=0, \quad \delta^{0}\left(M_{\Phi}\right) \phi_{0}=0 .
$$

Proof. That $M_{\Phi} \in L^{2}\left(\mathbb{R}, \mathcal{L}\left((E), \mathcal{g}^{*}\right)\right)$ follows from Lemma 5.1. For any $\psi \in(E)$ we have

$$
\begin{aligned}
\left\langle\left\langle\delta^{+}\left(M_{\Phi}\right) \phi_{0}, \psi\right\rangle\right\rangle & =\left\langle\left\langle\mathcal{K} \delta^{+}\left(M_{\Phi}\right), \psi \otimes \phi_{0}\right\rangle\right\rangle=\left\langle\left\langle\mathcal{K} M_{\Phi},(\nabla \otimes I)\left(\psi \otimes \phi_{0}\right)\right\rangle\right\rangle \\
& =\int_{\mathbb{R}}\left\langle\left\langle\mathcal{K} M_{\Phi(t)},(\nabla \psi(t)) \otimes \phi_{0}\right\rangle\right\rangle d t=\int_{\mathbb{R}}\left\langle\left\langle M_{\Phi(t)} \phi_{0}, \nabla \psi(t)\right\rangle\right\rangle d t \\
& =\int_{\mathbb{R}}\langle\langle\Phi(t), \nabla \psi(t)\rangle\rangle d t=\langle\langle\delta(\Phi), \psi\rangle\rangle,
\end{aligned}
$$

which proves the first identity in (5.8). The second and third identities are obtained similarly. We need only to note that $\nabla \phi_{0}=0$.

\section{Acknowledgements}

This work was supported by the Korea-Japan Basic Scientific Cooperation Program (2007-2009) "Noncommutative Stochastic Analysis and Its Applications to Network Science" and was completed during the second author's visit to Chungbuk National University in January, 2009. He thanks Professor Ji for his kind hospitality.

\section{REFERENCES}

[1] Aase, K., Øksendal, B., Privault, N., and Ubøe, J., "White noise generalizations of the Clark-Haussmann-Ocone theorem with application to mathematical finance," Finance Stochast., 4: 465-496 (2000).

[2] Attal, S., and Lindsay, J. M., "Quantum stochastic calculus with maximal operator domains," Ann. Probab., 32: 488-529 (2004).

[3] Belavkin, V. P., “A quantum nonadapted Ito formula and stochastic analysis in Fock scale,” J. Funct. Anal., 102: 414-447 (1991).

[4] Benth, F. E., and Potthoff, J., "On the martingale property for generalized stochastic processes," Stoch. Stoch. Rep., 58: 349367 (1996).

[5] Chung, D. M., Ji, U. C., and Obata, N., "Quantum stochastic analysis via white noise operators in weighted Fock space," Rev. Math. Phys., 14: 241-272 (2002).

[6] Hida, T., Kuo, H.-H., Potthoff, J., and Streit, L., "White Noise: An Infinite Dimensional Calculus," Kluwer Academic Publishers (1993).

[7] Hudson, R. L., and Parthasarathy, K. R., "Quantum Ito's formula and stochastic evolutions," Commun. Math. Phys., 93: 301323 (1984).

[8] Ji, U. C., "Stochastic integral representation theorem for quantum semimartingales," J. Funct. Anal., 201: 1-29 (2003).

[9] Ji, U. C., and Obata, N., Quantum white noise calculus, in "Non-Commutativity, Infinite-Dimensionality and Probability at the Crossroads (Obata, N., Matsui, T., and Hora, A., Eds.), pp. 143-191, World Scientific (2002).

[10] Ji, U. C., and Obata, N., "A role of Bargmann-Segal spaces in characterization and expansion of operators on Fock space," J. Math. Soc. Japan, 56: 311-338 (2004).

[11] Ji, U. C., and Obata, N., Admissible white noise operators and their quantum white noise derivatives, in "Infinite Dimensional Harmonic Analysis III (Heyer, H., Hirai, T., Kawazoe, T., and Saito, K., Eds.),” pp. 213-232, World Scientific (2005).

[12] Ji, U. C., and Obata, N., "Annihilation-derivative, creation-derivative and representation of quantum martingales," Commun. Math. Phys., 286: 751-775 (2009).

[13] Ji, U. C., and Obata, N., Quantum stochastic integral representations of Fock space operators, Stochastics, 81: 367-384 (2009).

[14] Kuo, H.-H., "White Noise Distribution Theory," CRC Press (1996).

[15] Lindsay, J. M., "Quantum and non-causal stochastic integral,” Probab. Theory Related Fields, 97: 65-80 (1993).

[16] Malliavin, P., "Stochastic Analysis," Springer-Verlag (1997).

[17] Meyer, P.-A., "Quantum Probability for Probabilists," Lect. Notes in Math. Vol. 1538, Springer-Verlag (1993).

[18] Nualart, D., "The Malliavin Calculus and Related Topics," Springer-Verlag, New York (1995).

[19] Obata, N., "White Noise Calculus and Fock Space," Lect. Notes in Math. Vol. 1577, Springer-Verlag (1994).

[20] Obata, N., "Generalized quantum stochastic processes on Fock space," Publ. RIMS, Kyoto Univ., 31: 667-702 (1995).

[21] Parthasarathy, K. R., "An Introduction to Quantum Stochastic Calculus," Birkhäuser (1992).

[22] Treves, F., “Topological Vector Spaces, Distributions and Kernels," Academic Press (1967). 